\title{
Self-Monitoring of Blood Glucose
}

\author{
Satish K. Garg ${ }^{1}$ and Irl B. Hirsch ${ }^{2}$
}

\section{Introduction}

A elf-monitoring of Blood Glucose (SMBG) continues to be an integral part of day-to-day diabetes management, especially in the emerging economies. Most of the Western world (United States and Western Europe) has been rapidly adopting continuous glucose monitoring (CGM) and is in the process of phasing out selfmonitoring of blood glucose, especially in patients with insulin-requiring diabetes (all patients with type 1 diabetes [T1D] and about $25 \%$ of patients with type 2 diabetes [T2D]). Due to the cost issues with the adoption of CGM, SMBG will continue to be used in emerging economies on a limited basis. The International Diabetes Federation has recommended the use of SMBG for both patients with T1D and T2D. The recent 5-year follow-up T1D Exchange data continues to show a direct correlation with glycated hemoglobin (HbA1c) levels and frequency of SMBG and increasing use of CGM. The key to improving outcomes for patients using SMBG relies on clinical decision support systems, which in turn will reduce diabetes burdens by guiding patients to manage their diabetes on a day-to-day basis. In this article, we review abstracts that highlight the challenges in SMBG, the need for behavioral change while using SMBG, and the role of clinical decision support systems

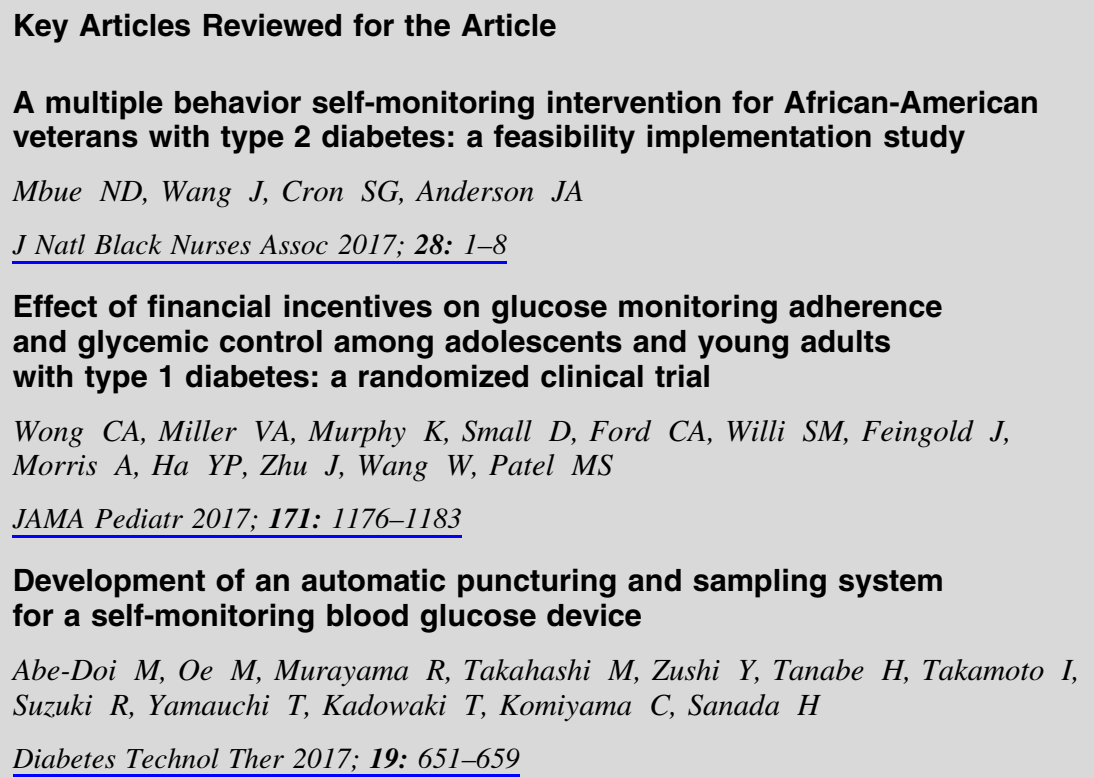

${ }^{1}$ University of Colorado Denver, Aurora, CO

${ }^{2}$ University of Washington School of Medicine, Seattle, WA 
Rational use of blood glucose test strips for self-monitoring in patients with diabetes mellitus: economic impact in the Portuguese healthcare system

Risso T, Furtado $C$

Diabetes Res Clin Pract 2017; 134: 161-167

Development of a protocol for automated glucose measurement transmission used in clinical decision support systems based on the continua design guidelines

Meyer M, Donsa K, Truskaller T, Frohner M, Pohn B, Felfernig A, Sinner F, Pieber T Stud Health Technol Inform 2018; 248: 132-139

Cost calculation for a flash glucose monitoring system for UK adults with type 1 diabetes mellitus receiving intensive insulin treatment

Hellmund R, Weitgasser R, Blissett $D$

Diabetes Res Clin Pract 2018; 138: 193-200

Evaluation of a new digital automated glycemic pattern detection tool

Comellas MJ, Albiñana E, Artes M, Corcoy R, Fernández-García D, García-Alemán J, García-Cuartero B, González C, Rivero MT, Casamira N, Weissmann J

Diabetes Technol Ther 2017; 19: 633-640

Proportion of daily capillary blood glucose readings required in the target range for target glycaemic control: shift of focus from target range to proportion in range

Sivasubramaniyam S, Amiel SA, Choudhary $P$

Diabet Med 2017; 34: 1456-1460

Postprandial dosing of bolus insulin in patients with type 1 diabetes: a cross-sectional study using data from the T1D exchange registry

Peters A, Van Name MA, Thorsted BL, Piltoft JS, Tamborlane WV

Endocr Pract 2017; 23: 1201-1209

Use of a connected glucose meter and certified diabetes educator coaching to decrease the likelihood of abnormal blood glucose excursions: the Livongo for Diabetes program

Downing J, Bollyky J, Schneider J

J Med Internet Res 2017; 19: e234

How satisfied are patients when their choice of funded glucose meter is restricted to a single brand?

Macdonald C, Lunt H, Downie M, Kendall D

J Diabetes Sci Technol 2017; 11: 1001-1006

Accuracy and user performance evaluation of a new, wireless-enabled blood glucose monitoring system that links to a smart mobile device

Bailey TS, Wallace JF, Pardo S, Warchal-Windham ME, Harrison B, Morin R,

Christiansen $M$

J Diabetes Sci Technol 2017; 11: 736-743

A multiple behavior self-monitoring intervention for African-American veterans with type 2 diabetes: a feasibility implementation study

Mbue $\mathrm{ND}^{1}$, Wang $\mathrm{J}^{2}$, Cron $S \mathrm{~S}^{2}$, Anderson $\mathrm{JA}^{1}$

${ }^{I}$ Health Sciences Research and Development Center for Innovations in Quality, Effectiveness and Safety, Houston, TX; Michael E. DeBakey Veterans Affairs Medical Center, Houston, TX; Baylor College of Medicine, Houston, TX;
${ }^{2}$ Center of Nursing Research, University of Texas Health Science Center at Houston School of Nursing, Houston, TX J Natl Black Nurses Assoc 2017; 28: 1-8

\section{Background}

This 3-month pretest-posttest study evaluated the feasibility of implementing a multiple-behavior self-monitoring intervention within a diabetes education program. 


\section{Methods}

Twenty African Americans $(n=20)$ attendees of a diabetes education classes at a large Veteran's Affairs hospital in Southwest Texas were selected for the study. Participants used either a smart phone application or paper diary to selfmonitor their blood glucose, diet, exercise, and weight.

\section{Results}

Using paired $t$ tests, patient self-monitoring of healthy lifestyle behaviors was found to be strongly related to improve blood glucose $(t=-3.858, P=0.001)$ and HbAlc $(t=-4.428, P<0.001)$, respectively. Spearman's correlation coefficient also showed significant associations between blood glucose and exercise ( $\mathrm{rs}=-0.68, P=0.008)$ as well as $\mathrm{HbAlc}$ and exercise $(\mathrm{rs}=-0.56, P=0.036)$.

\section{Conclusions}

In this feasibility study, multiple-behavior self-monitoring was shown to be effective in reducing blood glucose and HbA1c levels among African-American veterans; however, to validate these preliminary findings, a randomized controlled trial with a larger sample is required.

\section{Effect of financial incentives on glucose monitoring adherence and glycemic control among adolescents and young adults with type 1 diabetes: a randomized clinical trial}

Wong $C A^{1,2}$, Miller $V A^{3}$, Murphy $K^{4}$, Small $D^{2,5}$, Ford $\mathrm{CA}^{3}$, Willi $\mathrm{SM}^{6}$, Feingold $\mathrm{J}^{7}$, Morris $A^{8}$, Ha $Y P^{8}$, Zhu $\mathrm{J}^{2,8}$, Wang $W^{2,8}$, Patel $\mathrm{MS}^{2,8,9,10}$

${ }^{I}$ Department of Pediatrics, Duke Clinical Research Institute, Duke-Margolis Center for Health Policy, Duke University, Durham, NC; ${ }^{2}$ Leonard Davis Institute of Health Economics, Center for Health Incentives and Behavioral Economics at the University of Pennsylvania, Philadelphia, PA; ${ }^{3}$ Division of Adolescent Medicine, The Children's Hospital of Philadelphia, Perelman School of Medicine and University of Pennsylvania, Philadelphia, PA; ${ }^{4}$ Division of Pediatric Endocrinology, The Children's Hospital of Philadelphia, Philadelphia, PA; ${ }^{5}$ Department of Statistics, The Wharton School, University of Pennsylvania, PA; ${ }^{6}$ Division of Pediatric Endocrinology, The Children's Hospital of Philadelphia and University of Pennsylvania, Philadelphia, PA; ${ }^{7}$ Icahn School of Medicine at Mount Sinai, New York, NY; ${ }^{8}$ Perelman School of Medicine at the University of Pennsylvania, Philadelphia, PA; ${ }^{9}$ Department of Medicine, Crescenz Veterans Affairs Medical Center, Philadelphia, Pennsylvania, PA; ${ }^{10}$ Health Care Management, The Wharton School, University of Pennsylvania, Philadelphia, PA

JAMA Pediatr 2017; 171: 1176-1183

This manuscript is also discussed in the article on Diabetes Technology and Therapy in the Pediatric Age Group, page S-123.

\section{Background}

Glycemic control often deteriorates during adolescence and the transition to young adulthood for patients with type 1 diabetes. The inability to manage type 1 diabetes effectively during these years is associated with poor glycemic control and complications from diabetes in adult life. The purpose of this trial was to determine the effect of daily financial incentives on glucose monitoring adherence and glycemic control in adolescents and young adults with type 1 diabetes.

\section{Methods}

The Behavioral Economic Incentives to Improve Glycemic Control Among Adolescents and Young Adults with Type 1 Diabetes (BE IN CONTROL) study was an investigatorblinded, 6-month, two-arm randomized clinical trial conducted between January 22 and November 2, 2016, with 3-month intervention and follow-up periods. Participants were recruited from the Diabetes Center for Children at the Children's Hospital of Philadelphia and consisted of 90 adolescent and young adult patients (aged 14-20 years) with suboptimally controlled T1D (HbA1c > 8.0\%). Daily blood glucose monitoring was performed using a wireless glucometer, with a daily blood glucose monitoring goal for all patients of four or more checks per day with one or more level within the goal range $(70-180 \mathrm{mg} / \mathrm{dL})$. During the 3-month intervention period, participants were offered a \$60 monthly incentive via virtual account; every day of nonadherence to the monitoring goals resulted in a $\$ 2$ deduction from the account. This incentive was discontinued during the 3 month follow-up period. All analyses were by intention to treat. Change in HbAlc level at 3 months was the primary outcome. Secondary outcomes comprised compliance with glucose monitoring goals and change in HbAlc levels at 6 months.

\section{Results}

A total of 181 participants were screened, 90 (52 girls and 38 boys; mean age was $16.3 \pm 1.9$ years) of whom were randomly assigned to either the intervention $(n=45)$ or control $(n=45)$ arms. Participants in the intervention group exhibited significantly greater adherence to glucose monitoring goals during the incentive period than did the control group $(50.0 \%$ vs $18.9 \%$; adjusted difference $27.2 \%$ [95\% confidence interval $9.5 \%$ to $45.0 \%$ ]; $P=0.003$ ) but this difference did not continue in the follow-up period ( $15.3 \%$ vs $8.7 \%$; adjusted difference $3.9 \%$ [95\% CI $-2.0 \%$ to $9.9 \%$ ]; $P=0.20$ ). The change in $\mathrm{HbA1c}$ levels from baseline did not differ significantly between groups at either 3 months (adjusted difference $-0.08 \%$ [95\% CI $-0.69 \%$ to $0.54 \%$ ]; $P=0.80$ ) or 6 months (adjusted difference $0.03 \%$ [ $95 \% \mathrm{CI}-0.55 \%$ to $0.60 \%$ ]; $P=0.93$ ).

\section{Conclusions}

Daily financial incentives led to increased adherence to glucose monitoring goals during the incentive period in adolescents and young adults with T1D but did not significantly improve glycemic control.

\section{Development of an automatic puncturing and sampling system for a self-monitoring blood glucose device}

Abe-Doi $M^{1,2}$, Oe $M^{2}$, Murayama $R^{1,2}$, Takahashi $M^{2}$, Zushi $Y^{3}$, Tanabe $H^{1,3}$, Takamoto $I^{4}$, Suzuki $R^{4}$, Yamauchi $T^{4}$, Kadowaki $T^{4}$, Komiyama $C^{5}$, Sanada $H^{1,6}$

${ }^{1}$ Department of Advanced Nursing Technology, Graduate School of Medicine, The University of Tokyo, Tokyo, Japan; ${ }^{2}$ Global Nursing Research Center, The University of Tokyo, Tokyo, Japan; ${ }^{3}$ Terumo R\&D Center, Kanagawa, Japan; 
${ }^{4}$ Department of Diabetes and Metabolic Diseases, Graduate School of Medicine, The University of Tokyo, Tokyo, Japan; ${ }^{5}$ Department of Nursing, The University of Tokyo Hospital, Tokyo, Japan; ${ }^{6}$ Department of Gerontological Nursing/ Wound Care Management, Graduate School of Medicine, The University of Tokyo, Tokyo, Japan

Diabetes Technol Ther 2017; 19: 651-659

\section{Background}

Performing SMBG is a clinical challenge for elderly people with low dexterity. An all-in-one-type SMBG device that can simply and easily puncture the skin and monitor blood glucose would be useful for them. We developed an automatic skin-puncturing and blood-sampling (APS) system for an all-in-one type of SMBG device. The aims of this study were to determine whether the developed APS system, which has automatic puncturing, squeezing, and application functions, could provide sufficient blood sample volumes for SMBG and to determine the factors associated with failure in the use of the system by adult volunteers.

\section{Methods}

We investigated the success rate of obtaining a $0.8-\mu \mathrm{L}$ sample using the APS system and determined the factors associated with failure in 140 adult volunteers. Participant characteristics, induration of puncturing sites, and states of finger grip conditions were evaluated as factors contributing to puncturing failure. Participant characteristics, skin hydration, states of finger grip, skin elasticity of the finger pad, and blood flow were evaluated as factors of a squeezing failure.

\section{Results}

The success rate was $61.9 \%$. Puncturing failure was $21.6 \%$, and squeezing failure was $16.5 \%$. Factors associated with automatic puncturing failure were male sex, larger finger diameter, and thicker finger pad. The only factor in squeezing failure was lower peripheral skin temperature.

\section{Conclusion}

Improvement of the finger station groove shape to prevent ischemia and adjustment of the squeezing angle would be useful improvements for the all-in-one type of SMBG device intended for elderly people with decreased dexterity.

\section{Rational use of blood glucose test strips for self-monitoring in patients with diabetes mellitus: economic impact in the Portuguese healthcare system}

Risso $T^{l}$, Furtado $C^{1,2}$

${ }^{1}$ Information and Strategic Planning Directorate, InfarmedNational Authority of Medicines and Health Products, I.P., Lisbon, Portugal; ${ }^{2}$ National School of Public Health, Universidade NOVA de Lisboa, Portugal

Diabetes Res Clin Pract 2017; 134: 161-167

\section{Aims}

In insulin-treated patients, SMBG is important for diabetes management; however, its effectiveness in patients treated with oral glucose-lowering drugs alone is not fully supported by current evidence. The purpose of this report is to characterize the prescription patterns for blood glucose test strips (BGTS) in Portugal and to estimate the potential cost savings that could be realized from the rational use of BGTS.

\section{Methods}

A retrospective analysis of the Portuguese database of electronic medical prescriptions was conducted to assess patterns of BGTS prescription. Database searches were carried out for the period of January 1, 2016, through December 31,2016 , for prescriptions of insulin and other antidiabetics as well as the associated prescriptions of BGTS.

\section{Results}

During 2016, antidiabetic medications were prescribed for $894,637,82.7 \%$ of whom were prescribed oral glucoselowering drugs only. BGTS were prescribed to 456,179 patients overall and were more frequently prescribed for patients being treated with insulin. Still, $42.8 \%$ of patients treated with oral glucose-lowering drugs only were also prescribed BGTS. A large proportion of those individuals were prescribed antidiabetic drugs that carry a lower risk of causing hypoglycemia, but even so, >200 BGTS were prescribed per year. Several scenarios for a more rational use of BGTS were estimated to result in a current cost savings of up to $€ 9.5$ million per year.

\section{Conclusions}

More than one-third of patients treated with oral glucoselowering drugs only were also prescribed BGTS, despite burgeoning evidence of their limited effectiveness in this population, causing a substantial economic burden to the health-care system. With this in mind, rational use of BGTS should be encouraged in Portugal.

\section{Comments}

The small feasibility study in African-American veterans showed that multiple behavior self-monitoring implementation was effective in lowering blood glucose (BG) and improved glycemic control. The second study highlights the role of financial incentives among adolescent and young adults with T1D using SMBG. The study concluded that the glucose monitoring was maintained but without any significant improvement in glycemic outcomes, despite financial incentives. The third abstract emphasizes the importance of an automatic puncturing and sampling system for SMBG. The authors conclude that improvement of the finger station groove shape prevents ischemia and might be useful in elderly patients who have limited dexterity. The fourth study is from a large, public health database in Portugal. Blood glucose testing was prescribed in about $50 \%$ of the subjects on antidiabetic medications. They concluded that BG testing results in cost savings of about 9.5 million euros per year and thus encouraged the physicians to use SMBG in patients using hypoglycemic agents, especially on insulin. 
Development of a protocol for automated glucose measurement transmission used in clinical decision support systems based on the continua design guidelines

Meyer $M^{1}$, Donsa $K^{1}$, Truskaller $T^{1}$, Frohner $M^{2}$, Pohn $B^{2}$, Felfernig $A^{3}$, Sinner $F^{1}$, Pieber $T^{1,4}$

${ }^{1}$ HEALTH - Joanneum Research Forschungsgesellschaft mbH, Graz, Austria; ${ }^{2}$ University of Applied Sciences Technikum Wien, Vienna, Austria; ${ }^{3}$ Graz University of Technology, Graz, Austria; ${ }^{4}$ Medical University of Graz, Graz, Austria

Stud Health Technol Inform 2018; 248: 132-139

\section{Background}

Nearly all therapeutic interventions for T2D are derived from glucose measurements. Therefore, fast, accurate data transmission from glucose meter to clinical decision support systems (CDSSs) is vital for the management of T2D. The objective of this study was to develop a prototype protocol for automated transmission of glucose measurements based on the Continua Design Guidelines and to embed the protocol into a CDSS for health-care professionals.

\section{Methods}

Literature and market research were completed to investigate the state of the art and use that as the basis to develop, integrate, and validate a protocol for automated glucose measurement transmission in an iterative process.

\section{Results}

Research findings led investigators to develop a standardized glucose measurement transmission protocol using middleware. The interface description to communicate with the glucose meter was illustrated and embedded into a CDSS.

\section{Conclusion}

With the goal of devising a promising way to reduce medication errors and improve user satisfaction, a prototype of an interoperable transmission of glucose measurements was developed and implemented in a CDSS.

\section{Cost calculation for a flash glucose monitoring system for UK adults with type 1 diabetes mellitus receiving intensive insulin treatment}

Hellmund $R^{1}$, Weitgasser $R^{2,3}$, Blissett $D^{4}$

${ }^{1}$ Abbott Diabetes Care, Alameda, CA; ${ }^{2}$ Privatklinik WehrleDiakonissen Salzburg, Abteilung für Innere Medizin, Salzburg, Austria; ${ }^{3}$ Paracelsus Medizinische Privatuniversität, Salzburg, Austria; ${ }^{4}$ Device Access UK Ltd, Michelmersh, Hampshire, UK

\section{Diabetes Res Clin Pract 2018; 138: 193-200}

\section{Background}

An analysis of cost-effectiveness is used to consider flash glucose monitoring as a possible replacement for routine selfmonitoring of blood glucose (SMBG) in patients with type 1 diabetes mellitus (T1DM) using intensive insulin, from the perspective of the UK National Health Service (NHS).

\section{Methods}

Base-case cost was calculated using a value of 10 tests per day, in accordance with the maximum frequency of glucose monitoring recommended by the 2015 National Institute for Health and Care Excellence guidelines (4-10 tests per day). For scenario analyses, the SMBG testing frequency (5.6 per day) observed in the IMPACT clinical trial and flash monitoring testing rate (16 per day) observed in a real-world analysis were both considered. An additional scenario also considered severe hypoglycemia as a potential cost factor.

\section{Results}

The base-case annual cost per patient with flash monitoring was $£ 234$ (19\%) lower than the base-case calculated for routine SMBG (10 tests per day). In scenario analyses of flash monitoring vs SMBG, the annual per-patient cost of flash monitoring compared with 5.6 SMBG tests per day was $£ 296$ higher, while it was $£ 957$ lower when compared with 16 SMBG tests per day. The annual per-patient cost of severe hypoglycemia for flash monitoring users was estimated to be $£ 221$, compared with $£ 428$ for routine SMBG users (based on 5.6 tests/day), representing a $£ 207$ cost reduction.

\section{Conclusions}

The flash monitoring system has a modest effect on the UK NHS's glucose monitoring costs for patients with T1DM using intensive insulin treatment. For people requiring frequent BG tests, flash monitoring may be cost-effective, especially when potential decreases in the rate of severe hypoglycemia are considered.

\section{Evaluation of a new digital automated glycemic pattern detection tool}

Comellas $M J^{1}$, Albiñana $E^{2}$, Artes $M^{3}$, Corcoy $R^{4,5}$, Fernández-García $D^{6}$, García-Alemán $J^{6}$,

García-Cuartero $B^{7}$, González $C^{4,5}$, Rivero $M T^{8}$, Casamira $N^{l}$, Weissmann $J^{9}$

${ }^{1}$ Roche Diabetes Care Spain SL, Barcelona, Spain; ${ }^{2}$ Vithas Hospital Internacional Medimar, Paediatrics Unit, Alicante, Spain; ${ }^{3}$ Adelphi Spain, Barcelona, Spain; ${ }^{4}$ Hospital de la Santa Creu i Sant Pau, Endocrinology and Nutrition Department, Medicine Department, Universitat Autònoma de Barcelona, Barcelona, Spain; ${ }^{5}$ CIBER-BBN, Zaragoza, Spain; ${ }^{6}$ Hospital Universitario Virgen de la Victoria, Endocrinology and Nutrition Unit, Málaga, Spain; ${ }^{7}$ Hospital Universitario Ramón y Cajal, Pediatric Endocrinology and Diabetes Unit, Madrid, Spain; ${ }^{8}$ Complejo Hospitalario Universitario de Ourense, Endocrinology and Nutrition Unit, Ourense, Spain; ${ }^{9}$ Roche Diabetes Care Deutschland GmbH, Mannheim, Germany

Diabetes Technol Ther 2017; 19: 633-640

\section{Background}

Blood glucose meters are reliable devices for data collection, providing electronic logs of historical data that are 
easier to interpret than handwritten logbooks. Automated tools to analyze these data are necessary to facilitate glucose pattern detection and to support treatment adjustment. These tools emerge in a broad variety and a more-or-less nonevaluated manner. This study compares eDetecta, a new automated pattern detection tool, with nonautomated pattern analysis in terms of time investment, data interpretation, and clinical utility, with the overarching goal to identify areas of improvement and potential safety risks early in the development and implementation of the tool.

\section{Methods}

A multicenter, web-based evaluation was used for the for data collection. Thirty-seven endocrinologists were invited to assess glycemic patterns of four real reports (two continuous subcutaneous insulin infusion [CSII] and two multiple daily injection [MDI]). Endocrinologist and eDetecta analyses were compared on time spent to analyze each report and agreement on the presence or absence of defined patterns.

\section{Results}

The eDetecta module noticeably reduced the time taken to analyze each case on the basis of the emminens eConecta reports (CSII, $18 \mathrm{~min}$; MDI, $12.5 \mathrm{~min}$ ), compared with the automatic eDetecta analysis. Agreement between endocrinologists and eDetecta varied depending on the patterns, with a high level of agreement in patterns of glycemic variability. Further analysis of low level of agreement led to identifying areas where algorithms used could be improved to optimize trend pattern identification.

\section{Conclusion}

eDetecta was a useful tool for glycemic pattern detection, helping clinicians to reduce time required to review emminens eConecta glycemic reports. No safety risks were identified during the study.

\section{Comments}

Clinical decision support systems are important, as explained by the first and third abstracts. The fast and accurate transfer of data from glucose meters to CDSSs was shown to be effective in managing T2D, and the data showed significant reduction in medication errors as well as improved user satisfaction. The electronic logs of historical data are easier to interpret than hand-written logbooks. The eDetecta module, developed by Roche Diagnostics, markedly reduced the time required to analyze data in patients using pumps or MDIs. A study sponsored by UK NHS compared the cost of SMBG vs use of flash glucose monitoring (FGM) in patients with T1D. The study compared the use of SMBG 5.6 times per day or 10 tests per day vs FGM. There was a significant annual cost saving per patient using FGM in all scenarios. Part of the cost savings was related to reduction and early detection in severe hypoglycemia. The study concluded that the use of FGM is cost saving for insulin-requiring patients with T1D and is approved for use in the UK.

\section{Proportion of daily capillary blood glucose readings required in the target range for target glycaemic control: shift of focus from target range to proportion in range}

Sivasubramaniyam $S^{1,2}$, Amiel $S A^{1,3}$, Choudhary $P^{1,3}$

${ }^{1}$ King's College London, London, UK; ${ }^{2}$ London North West Healthcare Trust, London, UK; ${ }^{3}$ King's College Hospital, London, UK

Diabet Med 2017; 34: 1456-1460

\section{Background}

Most patients with T1D follow guidelines regarding target pre- and post-meal capillary blood glucose (CBG) levels to reach optimal glycemic control. We investigated the proportion of daily CBG readings between 4 and $10 \mathrm{mmol} / \mathrm{L}$ in people achieving different levels of glycemic control, as measured by HbA1c levels.

\section{Method}

Participants included 201 adults with T1D who were being treated with continuous subcutaneous insulin infusion (CSII) at one hospital clinic. Routine data were downloaded from each patient's insulin pump/meter for analysis. Patients were excluded for the following reasons: CSII $<6$ months, $<3 \mathrm{CBG} /$ day, pregnancy, hemoglobinopathy, and continuous sensor use. Participants were categorized into three groups based on HbAlc: $<58 \mathrm{mmol} / \mathrm{mol},<7.5 \%(\mathrm{n}=58) ; 58-74 \mathrm{mmol} / \mathrm{mol}$, $7.5 \%-8.9 \%(\mathrm{n}=107)$; and $\geq 75 \mathrm{mmol} / \mathrm{mol}, \geq 9.0 \%(\mathrm{n}=36)$.

\section{Results}

Mean age of participants was $43 \pm 13$ years, and mean HBA1c was $64 \mathrm{mmol} / \mathrm{mol}(8.0 \pm 1.1 \%)$. Reasons for starting CSII were raised HbA1c (47\%) and hypoglycemia (25\%), with the remainder (28\%) starting during pregnancy. Meter/pump data downloads contained a mean of $22 \pm 6.8$ days of data per participant. CBG testing frequency was similar between the three groups $(5.6 \pm 2.0,5.6 \pm 1.9$, and $5.4 \pm 1.2 \mathrm{CBG}$ readings/ day, respectively; $P=0.468$ ). The proportion of $\mathrm{CBG}$ readings within the target range of 4 and $10 \mathrm{mmol} / \mathrm{L}(72-180 \mathrm{mg} / \mathrm{dL})$ was $57.3 \pm 25.4 \%, 50.6 \pm 11.1 \%$, and $39.9 \pm 16.5 \%(P<0.0001)$. The proportion with $\mathrm{CBG}<4 \mathrm{mmol}$ was $13.8 \%, 8.8 \%$, and 4.4\% $(P<0.0001)$, respectively; and the proportion of readings $>10 \mathrm{mmol} / \mathrm{L}$ was $28.9 \pm 16.5 \%, 40.6 \pm 12.1 \%$, and $55.6 \pm 17.9 \%$ $(P<0.0001)$ in the three groups, respectively.

\section{Conclusions}

In participants who achieved HBA1c $<58 \mathrm{mmol} / \mathrm{mol}$ $(<7.5 \%) \sim 60 \%$ of CBG readings were within target range $(4-10 \mathrm{mmol} / \mathrm{L})$, with as much as $30 \%$ of readings $>10 \mathrm{mmol} /$ L. This goal of reaching $60 \%$ or more CBG readings within target range and being permissive with up to $30 \%$ readings higher than the target $(>10 \mathrm{mmol} / \mathrm{L})$ may represent a novel target for patients with diabetes and may reduce anxiety associated with levels that are occasionally out of range.

\section{Comments}

Now that we are using time in range as the fundamental metric for CGM data, it seems reasonable to categorize 
capillary blood glucose data in these same "buckets" of time-in-range, time-above-range, and time-below-range. Ideally, future studies can confirm how before-meal and bedtime glucose levels in each bucket correlate to HbA1c ranges, so patients can have specific goals.

\section{Postprandial dosing of bolus insulin in patients with type 1 diabetes: a cross-sectional study using data from the T1D exchange registry}

Peters $A^{1}$, Van Name $M A^{2}$, Thorsted $B L^{3}$, Piltoft $J S^{3}$, Tamborlane $W V^{2}$

${ }^{1}$ Keck School of Medicine, University of Southern California, Los Angeles, CA; ${ }^{2}$ Department of Pediatrics, Yale University School of Medicine, New Haven, CT; ${ }^{3}$ Novo Nordisk A/S, Bagsvard, Denmark

Endocr Pract 2017; 23: 1201-1209

\section{Objective}

A cross-sectional study was conducted to assess the prevalence and characteristics of patients with type 1 diabetes (T1D) who dose bolus insulin postprandially (PostP) vs preprandially (PreP).

\section{Methods}

Data were obtained from the enrollment questionnaires of 21,533 individuals in the T1D Exchange Registry. Patients were classified as PreP if they dosed "immediately before meal" or "several minutes before meal" were classified as PreP. Those who dosed "during meal" or "after meal" were classified as PostP. Data (PostP vs PreP) are reported as mean \pm standard deviation and percentage, as appropriate.

\section{Results}

After excluding patients who failed to answer the dosetiming question along with those who selected "not given regularly" or "depends on glucose level prior to meal" (4229 of 25,762 patients considered for the study), 21,533 patients were selected for inclusion. Nearly all patients (99\%) used rapid-acting insulin analogues, while 32\% dosed insulin PostP. Young patients (children $<18$ years of age) who dosed PostP vs those dosing PreP were characterized by higher HbA1c $(8.7 \pm 1.5 \% \quad[72 \pm 16.4 \mathrm{mmol} / \mathrm{mol}] \quad$ vs $8.4 \pm 1.7 \% \quad[68 \pm$ $18.6 \mathrm{mmol} / \mathrm{mol}])$; larger insulin dose $(1.2 \pm 0.7 \mathrm{IU} / \mathrm{kg} /$ day vs $1.1 \pm 0.7 \mathrm{IU} / \mathrm{kg} /$ day); more frequent history of hypoglycemia; and diabetic ketoacidosis. Adults who used PostP vs PreP dosing were younger $(33.0 \pm 15.3$ years vs $39.5 \pm 16.6$ years $)$, with higher HbAlc $(8.3 \pm 1.5 \%$ [67 $\pm 16.4 \mathrm{mmol} / \mathrm{mol}]$ vs $7.8 \pm 1.5 \%[62 \pm 16.4 \mathrm{mmol} / \mathrm{mol}])$ and larger insulin dose $(1.0 \pm 0.6 \mathrm{IU} / \mathrm{kg} /$ day vs $0.9 \pm 0.5 \mathrm{IU} / \mathrm{kg} /$ day $)$.

\section{Conclusion}

The results of this study reveal that a large proportion of patients use PostP dosing of bolus insulin. Even when using current rapid-acting insulin analogues, patients across all age groups who dose PostP are characterized by poorer glycemic control, and children who do so exhibit a greater prevalence of history of severe hypoglycemia and DKA.

\section{Comments}

The most surprising piece of information is how frequently mealtime insulin is injected during or after the meal. For both children and adults, about one-third of patients give their insulin after the recommended time. Not surprisingly, almost half of children less than 6 years of age were given their insulin after eating. One has to wonder for the entire population, but especially the children, how many who gave their insulin after eating actually would forget the injection, resulting in more hyperglycemia (and ketoacidosis), and how many physicians increased insulin dosing based on glucose data, not knowing the timing of the insulin administration. It is easy to see that if this higher insulin dose is subsequently injected prior to eating, hypoglycemia could occur.

\section{Use of a connected glucose meter and certified diabetes educator coaching to decrease the likelihood of abnormal blood glucose excursions: the Livongo for Diabetes program}

\section{Downing $J^{1}$, Bollyky $J^{2,3}$, Schneider $J^{2}$}

${ }^{1}$ Center of Health and Community, University of California, San Francisco, San Francisco, CA; ${ }^{2}$ Livongo Health, Mountain View, CA; ${ }^{3}$ Department of Medicine, Stanford University Medical Center, Stanford, CA

J Med Internet Res 2017; 19: e234

This manuscript is also discussed in the article on Using Digital Health Technology to Prevent and Treat Diabetes, page S-79.

\section{Background}

The Livongo for Diabetes Program offers members (1) a blood glucose (BG) monitoring device that is cellular technology-enabled with two-way messaging, which centrally stores the BG information and delivers real-time messages back to the individual; (2) unlimited supply of glucose test strips; and (3) access to a team of diabetes lifestyle coaches who can answer questions and assist with goal setting, as well as automated support for abnormal glucose excursions. The program is sponsored by at-risk self-insured employers, health plans, and provider organizations that offer it free of cost to members with diabetes. The program is also available directly to self-paying patients with diabetes. This study evaluates BG data from 4544 individuals with diabetes who were enrolled in the Livongo program from October 2014 through December 2015.

\section{Methods}

Members measured BG levels using the Livongo glucose meter (1.8 times per day on average). Using individual fixed effects to control for individual characteristics, we estimated the probability of a patient having a day in which a BG reading is outside of the normal range $(70-180 \mathrm{mg} / \mathrm{dL}$, or $3.9-$ $10.0 \mathrm{mmol} / \mathrm{L}$ ) during months $2-12$ vs month 1 of the program.

\section{Results}

Livongo members realized an average decrease of $18.4 \%$ in the likelihood of having a day with hypoglycemia (BG $<70 \mathrm{mg} /$ $\mathrm{dL}$ ) in months $2-12$ compared with month 1 as the baseline, as 
well as a $16.4 \%$ average decrease in hyperglycemia (BG $>180 \mathrm{mg} / \mathrm{dL})$. The biggest improvement was seen in hyperglycemia among members who did not use insulin. We do not know all of the contributing factors such as medication or other treatment changes during the study period.

\section{Conclusions}

Our findings suggest that patients having access to a connected glucose meter and certified diabetes educator coaching are associated with decreased likelihood of abnormal glucose excursions, an improvement which could lead to lower diabetes-related health-care costs.

\section{Comments}

The concept of a two-way messaging device that can communicate with a diabetes education team, with the ultimate goal of improving glycemic control and reduce costs, is a novel idea that deserves testing. Unfortunately, this was not a true randomized controlled trial, and as the authors acknowledge, it is impossible to know what was responsible for the improved glucose levels. It is also difficult to make any conclusions since the timing of the fewer than two glucose tests per day could have been biased for a variety of reasons. Without knowing $\mathrm{HbA} 1 \mathrm{c}$ levels and with this amount of glucose testing, it is difficult to know whether glucose excursions, hypoglycemia, or any aspect of glucose control were improved. Still, the concept is important for the millions of people with diabetes with minimum access to diabetes educators. Ideally, a true randomized trial with a control group is being planned, which will also capture short- and long-term costs.

\section{How satisfied are patients when their choice of funded glucose meter is restricted to a single brand?}

Macdonald $C^{1}$, Lunt $H^{2,3}$, Downie $M^{4}$, Kendall $D^{3}$

${ }^{I}$ Department of General Medicine, Christchurch Hospital, Christchurch, New Zealand; ${ }^{2}$ Department of Medicine, University of Otago Christchurch, Christchurch, New Zealand; ${ }^{3}$ Diabetes Centre, Christchurch Hospital, Christchurch, New Zealand; ${ }^{4}$ Department of Endocrinology, Southland Hospital, Invercargill, New Zealand

\section{J Diabetes Sci Technol 2017; 11: 1001-1006}

\section{Background}

A large number of governments and insurers are employing central management of purchasing decisions to reduce the cost of medical devices they cover, including glucose meters. We report patients' responses 1 year after an "enforced" change in brand of glucose meter to sole national supplier arrangement for funded glucose meters and strips.

\section{Method}

One year after the final changeover date to a single glucose meter supplier, specialist diabetes clinic attendees from two geographical locations completed a questionnaire regarding the change. The first location asked consecutive patients at the facility to complete a glucose meter satisfaction ques- tionnaire during their visit. The second clinic location mailed the same questionnaire to attendees. Patients' responses to open questions were analyzed thematically.

\section{Results}

The first clinic location had an $85 \%$ response rate, while the rate for the second location was $31 \%$. A total of 378 questionnaires (309 from the first and 69 from the second) were deemed suitable for analysis, and $90 \%$ of included participants were insulin users. Results from the two locations were broadly similar. Most patients adapted well to the meter changeover, although $36 \%$ of respondents reported ongoing dissatisfaction with the "new" meter. Meter accuracy and precision was the most commonly cited concern (23\% of participants).

\section{Conclusions}

One year after glucose meter changeover, approximately one-third of participating patients expressed dissatisfaction with the provider-selected meter, and many participants reporting their failure in adapting to the sole supplier scheme. Allowing patients to select their meters and strips, ideally from a choice of two or more brands that vary in technical and ergonomic features, is likely to result in higher overall patient satisfaction than is a sole supplier arrangement.

\section{Comments}

We are now approaching 40 years of home blood glucose testing. All would agree it revolutionized diabetes management, particularly for those receiving insulin therapy. The early meters were large, clumsy, and not very accurate. Over time they improved in all aspects, including ergonomics, convenience, and accuracy/precision. With glucose testing becoming the standard of care, meters and strips became commoditized, yet many patients had clear preferences of one meter compared with another. Unfortunately, the business evolved so that payers had economic incentives to use the glucose testing system with the lowest cost. This is really no different than any other aspect of the business of medicine, and thus it is not surprising that when patients lose their choice of which meter to use, some are quite dissatisfied. The greatest concern is perceived inaccuracies, which many recent studies have shown to be valid. It is hoped that this aspect is taken into consideration by the payers. What we know, however, is that it rarely is.

\section{Accuracy and user performance evaluation of a new, wireless-enabled blood glucose monitoring system that links to a smart mobile device}

Bailey $\mathrm{TS}^{1}$, Wallace $\mathrm{JF}^{2}$, Pardo $\mathrm{S}^{2}$, Warchal-Windham $\mathrm{ME}^{2}$, Harrison $B^{2}$, Morin $R^{2}$, Christiansen $M^{3}$

${ }^{1} A M C R$ Institute Inc., Escondido, CA; ${ }^{2}$ Ascensia Diabetes Care, Parsippany, NJ; ${ }^{3}$ Diablo Clinical Research, Walnut Creek, CA.

\section{J Diabetes Sci Technol 2017; 11: 736-743}

\section{Background}

The ContourPlus ONE is a new blood glucose monitoring system (BGMS) with a user-friendly, wireless- and 
Bluetooth-enabled blood glucose meter that can be linked to a smart mobile device and sync with the Contour Diabetes app on a smartphone or tablet.

\section{Methods}

In compliance with the International Organization for Standardization (ISO) 15197:2013 criteria for in vitro diagnostic test systems, two studies were carried out to assess the accuracy of this new BGMS. Study 1 (the laboratory study) tested fingertip capillary blood samples from 100 subjects. Tests were run in duplicate using test strip from three different lots. Study 2 (clinical study) included 134 subjects with T1D or T2D from clinical sites. BGMS results were compared with YSI analyzer (YSI) reference results for fingertip blood obtained for selftesting by untrained subjects and for subject fingertip, palm, and venous samples obtained by trained study staff.

\section{Results}

In study 1 , of the combined results for all three test strip lots, $99.0 \%(594 / 600)$ fulfilled accuracy criteria set forth in ISO 15197:2013 Section 6.3. In study 2, 99.2\% (133/134) of subjectobtained capillary fingertip results and 99.2\% (133/134) of study staff-obtained fingertip results met ISO 15197:2013 Section 8 accuracy criteria. Similarly, 99.2\% (125/126) of results from subject-obtained palm samples and 100\% (132/132) from study staff-obtained venous samples in study 2 met the ISO accuracy criteria. Moreover, 95.5\% (128/134) of subject-obtained fingertip self-test results were within $\pm 10 \mathrm{mg} / \mathrm{dL}( \pm 0.6 \mathrm{mmol} / \mathrm{L})$ or $\pm 10 \%$ of the YSI reference result. Questionnaire responses indicate that most participants found the BGMS easy to use.

\section{Conclusions}

The BGMS exceeded ISO 15197:2013 accuracy criteria in both laboratory testing and in a clinical setting when used by untrained subjects with diabetes.

\section{Comments}

The smartphone has become the center of our growing technology-filled world. It seems that use of an integrated app should be standard for diabetes management, particularly for insulin-users. Tracking of blood glucose levels (fingerstick or CGM), insulin dosing (perhaps with a bolus calculator), exercise, sleep, and suggestions/ coaching can all be accomplished using a phone. The need to put all of these elements into one app is slowly occurring.

\section{Conclusions}

As we approach 40 years of home blood glucose monitoring, several conclusions can be made. First, despite vast improvements in the technology, many available meters still do not meet current standards for accuracy. Cost drives much of this, but unfortunately, this problem has not resolved. On the other hand, the use of newer technologies such as cloud-based storage of data with real-time coaching from educators is now a reality. The use of the smartphone app is becoming more common, and both patients and their physicians should have an easier time assessing the data. And while CGM has become the standard of care for many, fingerstick home blood glucose monitoring will remain in those countries that can't afford the more expensive technology. The concern, which we are already seeing, is that innovation for the home blood glucose meters will not progress, as industry sees more rewards with CGM. Our major hope at this time is that the quality of our glucose strips and meters do not suffer.

\section{Author Disclosure Statement}

No competing financial interests exist for this manuscript. 